\title{
A combined optical, SEM and STM study of growth spirals on the polytypic cadmium iodide crystals ${ }^{\dagger}$
}

\author{
RAJENDRA SINGH, S B SAMANTA ${ }^{*}$, A V NARLIKAR * and G C TRIGUNAYAT \\ Department of Physics and Astrophysics, Delhi University, New Delhi 110 007, India \\ ${ }^{\ddagger}$ National Physical Laboratory, Dr K S Krishnan Road, New Delhi 110 012, India
}

\begin{abstract}
Some novel results of a combined sequential study of growth spirals on the basal surface of the richly polytypic $\mathrm{CdI}_{2}$ crystals by optical microscopy, scanning electron microscopy (SEM) and scanning tunneling microscopy (STM) are presented and discussed. In confirmation of the known structural data, the STM pictures clearly reveal the value of unit cell $a(=b)$ dimension to be equal to $0.428 \mathrm{~nm} a$ and the value of the interaxial angle $\gamma$ to be equal to $120^{\circ}$. Under the high resolution and magnification achieved in the scanning electron microscope, the growth steps of large heights seen in the optical micrographs are found to have a large number of additional steps of smaller heights existing between any two adjacent large height growth steps. When further seen by a scanning tunneling microscope, which provides still higher resolution, several unit substeps are seen to exist between two consecutive additional steps. The height of each substep is found to be equal to the unit cell height of the underlying polytype. The height of the larger additional steps are found to be integral multiple of the unit cell height. The horizontal distance between two consecutive growth steps is found to be an integral multiple of the lattice parameter $a$. The interspacing of the growth steps of same step height is found to vary at different positions on the same crystal face.
\end{abstract}

Keywords. $\mathrm{CdI}_{2}$; polytypism; scanning tunneling microscopy (STM), spiral step height; dislocation.

\section{Introduction}

Cadmium iodide is known to be a richly polytypic material. Several hundreds of $\mathrm{CdI}_{2}$ polytypes have been discovered, of which complete crystal structure of nearly 200 polytypes have been determined (Trigunayat 1991). Growth spirals are frequently observed on the basal surfaces of the $\mathrm{CdI}_{2}$ crystals. The heights of the spiral steps had been measured by Forty (1952), Trigunayat and Verma (1962) and Chadha and Trigunayat (1966), employing the method of internal interference of light. However, later Tubbs (1971) raised doubt about correctness of the reported step height values, as the method of internal interference involved an accurate knowledge of the value of refractive index of the material, which was found to be controversial.

The technique of scanning tunneling microscopy (STM) enables observation of surface features at magnification and resolution much higher than with an optical or scanning electron microscopy (SEM). It also affords an accurate measurement on the growth features, e.g. height of a growth step or spacing between two consecutive steps. Thus, unlike the method of internal interference which is beset with uncertainty on account of ambiguity in the value of refractive index of the material of the crystal, the STM

\footnotetext{
*Author for correspondence

${ }^{\dagger}$ Paper presented at the 5th IUMRS ICA98, October 1998, Bangalore
}

furnishes an unambiguous and accurate measurement of step heights on the crystal surface. Several STM studies on the polytypic crystals of silicon carbide have been reported (Bonnell and Clarke 1988).

The present paper reports the results of a combined study involving optical microscopy, SEM, STM and Xray diffraction. They provide some unique precise information about heights of spiral steps, interspacing of the steps, bunching of steps and formation of growth spirals on the crystal surface.

\section{Experimental}

Cadmium iodide was highly purified by zone refining technique, employing eighteen zone passes, and then employed for crystal growth by slow evaporation of its aqueous solution in a petri dish. Most of the crystals grew as hexagonal platelets with shining (0001) basal faces, measuring 1-2 mm across and a few hundred $\mu$ in thickness. The crystals displaying well formed single growth spirals on their basal faces were selected for further investigation.

The crystals were first examined through an optical projection microscope in transmission mode and the growth spirals were photographed. Then they are subjected to X-ray diffraction for identification of their constituent polytypes. Toward this end, $15^{\circ}$ oscillation photograph of a crystal was taken with the crystal so positioned that a long succession of $10 \cdot 1$ reflections, 
which are particularly suited for polytype identification, were recorded on the zero layer line (Trigunayat and Verma 1962). Next, the crystals were gold coated for SEM and STM, to render the surface conducting. Finally, the basal surfaces of the crystals were examined through a STM, which also enabled accurate measurement of spiral step height as well as interstep spacing. A Nanoscope-II system STM was employed that enabled at least $0.01 \mathrm{~nm}$ accuracy of measurement. The instrument was routinely calibrated with a standard HOPG (highly oriented pyrolytic graphite) crystal, to ensure authenticity of the measured values.

\section{Results}

Crystals of the common $\mathrm{CdI}_{2}$ polytype $4 \mathrm{H}$ (hexagonal 4layered unit cell) were selected for the present investigation. The polytype identification was done by X-ray diffraction, as outlined in the previous section. According to the known standard crystallographic data for the polytype $4 \mathrm{H}$, the unit cell parameters are $a=b=0.428 \mathrm{~nm}$, $c=1.3728 \mathrm{~nm} ; \alpha=\beta=90^{\circ}, \gamma=120^{\circ}$, which found a pictorial atomic level confirmation in the STM observations.

Figure 1 depicts a single hexagonal growth spiral on the basal surface of a $4 \mathrm{H}$ crystal, as seen through an optical microscope. Well developed regularly spaced growth steps are seen on two hexagonal sides. When observed through a scanning electron microscope, additional substeps were found to exist between two successive main steps, as seen in figure 2. Twelve such substeps are seen between two main steps at the position indicated by double arrowmark in the figure. The steps were found to bunch together at several positions. The bunching appeared at regular intervals at some places, but at most other places it occurred randomly (shown by arrowmark

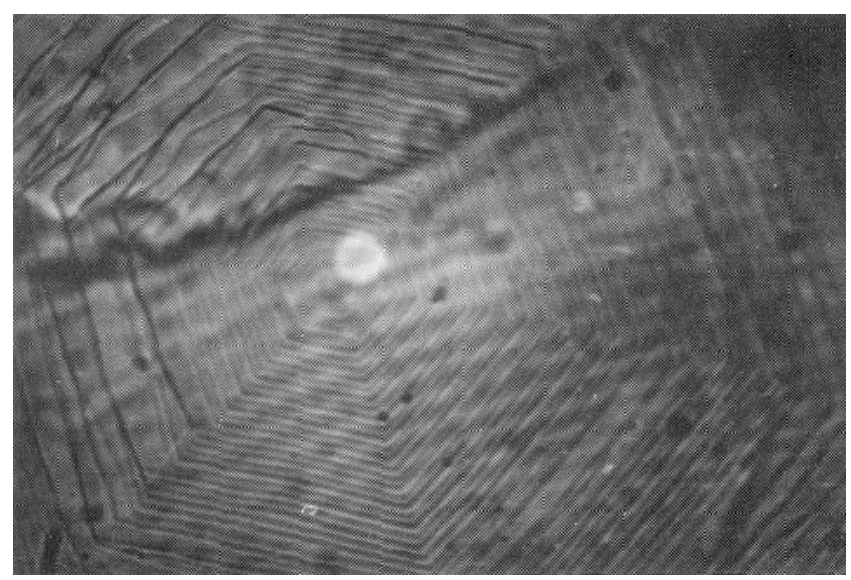

Figure 1. Optical micrograph of a hexagonal growth spiral on the basal surface of a crystal $(\times 370)$. The superimposed weak intensity broad fringes spuriously arise from two-beam interference of light in the optical system of the microscope. in figure 2). Such bunching is also seen to occur at several positions, particularly away from the centre, in the optical micrograph (figure 1).

Next the crystal surfaces were examined by STM. A linear profile of the variation of step height in the direction of scanning in two different regions of crystal surface is represented in figure 3, which shows a large succession of unit growth steps, with an intervening large step. Remarkably, under the extremely high resolution and magnification achieved in the STM, now even growth steps corresponding to the unit cell height of the underlying polytype $4 \mathrm{H}$ are clearly revealed. The vertical distances indicated in figure 3 do not represent actual step heights, which are found by adding a constant correction factor of $0.31 \mathrm{~nm}$, arising from the existence of weak van der Waals force between the crystal surface and the bottom of the overlying composite $4 \mathrm{H}$ layer (Singh et al 1998). The interstep spacing is $8.51 \mathrm{~nm}$ for the unit steps.

\section{Discussion}

The optical micrograph (figure 1) shows regularly spaced growth spiral steps of large height. The good visibility of the steps suggests the value of the step height to be a few tens of nm. When viewed under higher resolution with a scanning electron microscope, substeps of smaller height are seen to exist between two consecutive spiral steps (figure 2). At positions of relatively better visibility 12 such substeps can be counted between two successive main steps. They are regularly spaced. The main steps now appear to have been produced by a somewhat peri-

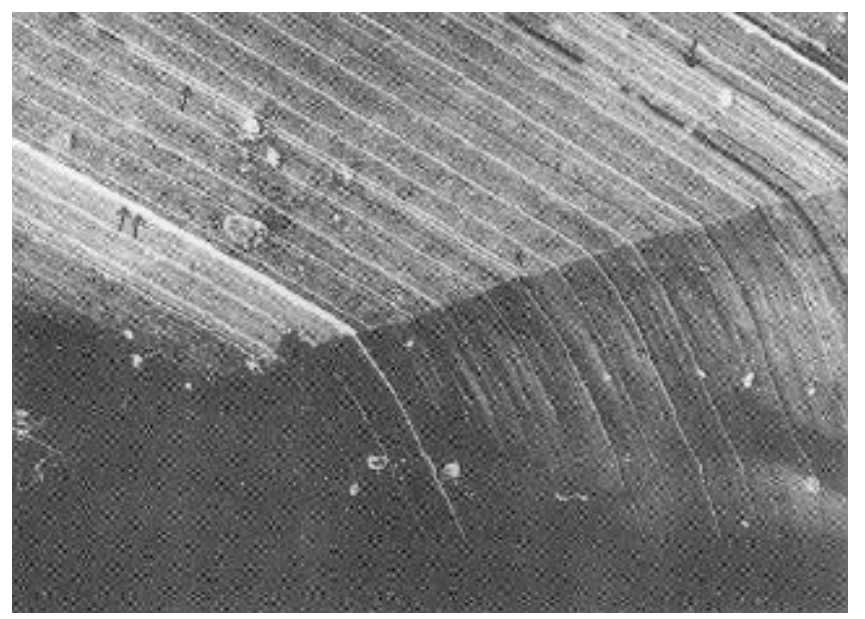

Figure 2. Scanning electron micrograph of a part of the spiral shown in figure 1 , revealing the existence of additional substeps between two consecutive main steps $(\times 1000)$. Twelve such substeps are seen between two main steps at the position indicated by double arrowmark in the figure. Bunching of growth steps at (a) regularly repeating positions (upwards pointing single arrowmarks) and (b) random positions (downwards pointing single arrowmarks) is seen. 


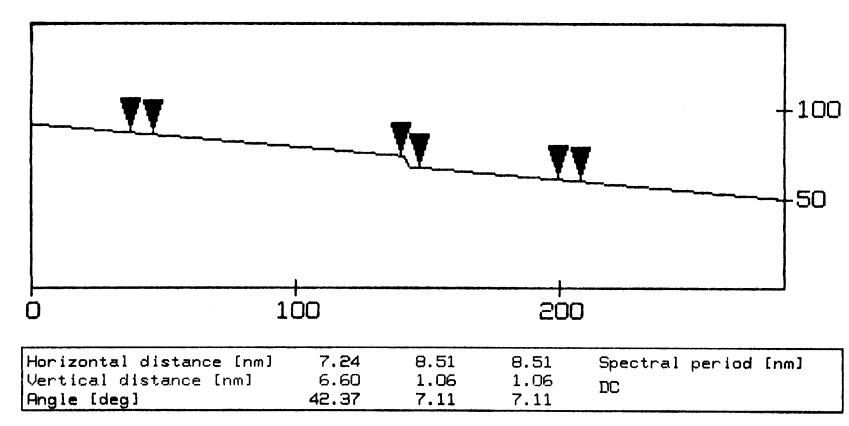

Figure 3. Scanning tunneling micrograph profile showing numerous unit growth steps and a bunched large height growth step.

odic bunching of the substeps. If the bunching occurs at a regularly repeating interval, nearly equispaced steps are produced (upwards pointing arrowmarks in figure 2). If the bunching is irregular, unevenly spaced steps are produced (downwards pointing arrowmarks in figure 2).

The following interferences may be drawn from these results. Initially a screw dislocation at large burgers vector strength would have emerged on the crystal surface, generating the growth spiral shown in figure 1, with well visible spiral steps. However, since a large dislocation is known to be thermodynamically unstable, it tends to decompose into smaller dislocations. Thus at some stage of crystal growth the original dislocation would have decomposed into 12 smaller dislocations, giving rise to 12 concentric spirals seen in figure 2. Here it is presumed that all the component dislocations are of the same hand and their mutual separation is less than $\rho_{c}$, the radius of the critical nucleus. Under these conditions the dislocations are known to 'cooperate' with one another to produce non-intersecting concentric growth spirals (Verma 1953). It should be noted that the initial growth spiral with large step height will not be rendered inoperative after the decomposition of the original dislocation. The smaller height growth steps pertaining to the 12 new spirals will periodically bunch at the edges of the steps of the original growth spiral, with a bunching period of 12 steps. Thus during the subsequent growth the steps belonging to both the old spiral and the 12 new spirals will continue to be simultaneously visible (figure 2). As time passes, the new dislocations will further tend to decompose into still smaller dislocations until the limit is reached when all the decomposed component dislocations have the burgers vector strength corresponding to the unit cell height of the polytype constituting the crystal, viz. the polytype $4 \mathrm{H}$. In other words one finally ends up with numerous concentric growth spirals due to unit dislocations. That is how the STM photograph (figure 3) shows a large succession of unit growth steps, corresponding to the cell height of the polytype 4H (Singh et al 1998). As expected, the interstep spacings represented as horizontal distances in figure 3, were all found to be integral multiple of the unit cell dimension $a$, although the values differed at different positions on the crystal surface.

\section{References}

Bonnell D A and Clarke D R 1988 J. Am. Ceram. Soc. 71629 Chadha G K and Trigunayat G C 1966 J. Crystal Growth C-18 313

Forty A J 1952 Philos. Mag. 43377

Singh R, Samanta S B, Narlikar A V and Trigunayat G C 1998 Surf. Sci. (to be published)

Trigunayat G C 1991 Solid State Ionics 483

Trigunayat G C and Verma A R 1962 Acta Crystallogr. 15499

Tubbs M R 1971 Acta Crystallogr. B27 857

Verma A R 1953 Crystal growth and dislocations (London: Butterworths Publications) Ch. 6 